\title{
Impact Force Reconstruction of Composite materials based on Improved Regularization Technology
}

\author{
Yajie Sun ${ }^{1,2,3^{*}}$, Tao Yin ${ }^{1}$, Jian Yang ${ }^{1}$, Zhiyu Cai ${ }^{1}$ and Shaoen $\mathrm{Wu}^{4}$ \\ ${ }^{1}$ School of Computer \& Software, Nanjing University of Information Science \& Technology, Nanjing, China \\ ${ }^{2}$ Engineering Research Center of Digital Forensics, Ministry of Education, Nanjing University of Information \\ Science \& Technology, Nanjing, China \\ ${ }^{3}$ Jiangsu engineering Centre of Network Monitoring, Nanjing University of Information Science \& Technology, \\ Nanjing, China \\ ${ }^{4}$ Department of Computer Science, Ball State University, Muncie, USA \\ [E-mail: yanacn@163.com, 2045945879@qq.com,1477178778@qq.com, 1037347598@qq.com, \\ swu1235@ilstu.edu] \\ ${ }^{*}$ Corresponding author: Yajie Sun
}

Received April 26, 2021; revised June 23, 2021; accepted July 31, 2021; published August 31, 2021

\begin{abstract}
In the structural health monitoring of composite materials, in order to solve the ill-posed problem of impact force reconstruction, regularization techniques are often used to deal with it. Due to the poor convergence of the traditional Tikhonov regularization method, in order to accurately reconstruct the time history of the impact force, this paper improves Tikhonov regularization method and constructs homotopy function with strong convergence. Since the optimal regularization parameters need to be found in the homotopy function, the Newton downhill method is used to find the optimal parameters and the homotopy function can be calculated, which can accurately reconstruct the time history of the impact force. In order to verify the universality of the method in this paper, impact hammers of different materials were used in the experiment in this paper to study and compare the reconstruction effect of impact time history of different impact hammers.
\end{abstract}

Keywords: Impact damage, composite materials, impact force reconstruction, improve regularization method. 


\section{Introduction}

In the actual service life of large mechanical equipment, a variety of structural damage often occurs under the action of various external forces, which damages its mechanical strength and ultimately leads to disastrous consequences [1-2]. Composite materials have outstanding potential and advantages in spacecraft manufacturing, the exploration of structural failures caused by external low-velocity shocks is very important for aerospace structures because these structures are often subjected to external forces during manufacture and operation. Accidental effects such as low-speed impacts from various external sources, including birds, hail, runway debris, fall tools, etc., can lead to serious damage, resulting in a decrease in stiffness, integrity and strength of the lightweight composite structure. Low-speed lateral impact can cause different types of damage, such as delamination of carbon fiber/epoxy laminate surfaces, matrix cracking, and fiber breakage in composite structures [3-4]. Therefore, the real-time monitoring of the impact force on the composite material is of great significance to judge the damage situation of the composite material and estimate the residual strength of the composite material [5]. By determining the position, direction and strength of the impact force, we can achieve a comprehensive understanding of the impact on the composite structure, and improve the structure design based on this. Mathematically, impact force recognition is an inverse problem [6-7].

The regularization methods for solving inverse problems have been widely used [8-9], including truncated singular decomposition, Tikhonov iteration, and basis function expansion [10-11]. Iterative regularization algorithms such as Landweber and conjugate gradient iterative regularization algorithm are different from the truncated singular value decomposition Tikhonov regularization method. There is no advantage of transferring matrix inversion and no need to clarify regularization parameters. The iterative process is regularized. The process is widely used in inverse problem solving but rarely used in force recognition. Wang Linjun [12] used the improved Tikhonov regularization method to identify different types of dynamic loads. The simulation example shows that it is superior to the Tikhonov regularization method.

Qiu et al. [13] used the time reversal method to study the identification of impact sources in a finite isotropic plate. For a given force position, it is found that the maximum peak of the reconstructed signal occurs at the impact location. Chen et al. [14] used the time reversal method to conduct experimental research on impact force recognition. In the experimental verification, a square aluminum plate with a piezoelectric sensor was used. The results show that the TR method eliminates the near-real-time monitoring of the ill-posedness and structure of the inverse problem. S. Atobe et al. [15] presented a method to identify impact force location and force history without using analytical model.

Kalhori $\mathrm{H}$ et al. [16-17] used the inverse algorithm to solve the impact damage problem of materials, Chein-Shan Liu and Chung-Lun Kuo [18] give a dynamical Tikhonov regularization method for solving nonlinear ill-posed problems and Li Jian et al. [19] gave a dynamical Newton-like method with adaptive step size for solving nonlinear algebraic equation, which provided a good foundation for this paper. Mustapha S et al. [20-21] studied the correlation coefficient between the original time reversal signal and the reconstruction time reversal signal to define the damage index of each sensing path, which is then used to identify multiple debonding during the fusion process.

In addition, the ill-conditioned problem of the inverse algorithm is also the focus of scholars at home and abroad. Rezghi $\mathrm{M}$ et al. [22] continued to improve and optimize the Tikhonov regularization algorithm for ill-conditioned problems. Chein-Shan Liu et al. [23] also gave a new regularization method to solve ill-posed linear systems. In the actual Lamb wave signal 
processing, the spatial resolution of the signal is an important factor affecting the resolution and precision of damage detection. In reference [24], the author proposed a signal domain transform method to transform the original dispersive Lamb wave signal from the time domain to the distance domain. The time-distance transformation on the excitation waveform was specially modified, and the spatial resolution of the signal was effectively improved. The team [25] also reconstructed a new linear dispersion signal from the original Lamb wave signal for dispersion compensation, and at the same time, the group velocity at the center frequency was reduced exponentially, so that the reconstructed signal could obtain good spatial resolution.

In the process of image denoising, Jianwei Zhang et al. [26] put forward an adaptive regularization parameter function based on the gradient and variance of the image, this method has good effect on edge and data denoising. Linjun Wang et al. [27] optimized the regularization method and applied it to practical engineering problems, which has a remarkable effect on solving dynamic force problems.

In this paper, the homotopy method constructor is selected and then the optimal solution is found by Newton's downhill method, and the homotopy function is obtained. The homotopy method has the advantages of universal validity, convergence and flexibility, and has good effects in image reconstruction. Therefore, it is applied to reconstruct the impact force time history. The Newton's downhill method not only has the advantage of Newton's iterative method, but also prevents the Newton's iterative method from being limited by the initial value selection. Therefore, this chapter uses Newton's downhill method to approximate the minimum value and find the optimal parameters. In the homotopy function, the optimal solution of regularization is obtained, that is, the optimal impact force size.

In this study, the first chapter describes the development of research methods in this direction. The second part of this paper, we introduce the related work of deconvolution and the traditional regularization method. The third part introduces the theory of the improved regularization method proposed in this paper. The fourth chapter summarizes the experimental equipment and summarizes the experimental process. The fifth chapter verifies the feasibility and accuracy of the method, and analyzes the experimental results.

\section{Previous Work}

\subsection{Deconvolution}

In general, the impact identification problem is non-linear because the expression involving system input, namely, the impact force and the system output, which is a function of unknown parameters [28]. For a better understanding, consider the material on which the force is applied. If the position of the applied force changes, the deflection of each point of the material will also change. Therefore, the identification of the impact location generally takes precedence over the estimation of the time history of the impact force.

In the vibration response of the linear system, for example, the dynamic strain $\varepsilon$ recorded at the point $\alpha$ caused by the impact force $f$ applied at the position $\beta$ can be expressed by the convolution integral as:

$$
\varepsilon(\beta, t)=\int_{0}^{t} k(\alpha, \beta, \mathrm{t}-\tau) f(\alpha, \tau) \mathrm{d} \tau
$$

Where, the transfer function $k(\alpha, \beta, t-\tau)$ is defined as an impulse response function at the point $\beta$ at which the impact force is applied at the point $\alpha$ at time $t=\tau$. The discrete form of convolution integral $\left(t_{i}=i \Delta t ; i=1, \ldots, p\right.$, where $p$ is the number of samples) can be 
represented by a Riemann approximation and written as a system of algebraic equations such as:

$$
k f=\varepsilon, k \in R^{m \times n}, f \in R^{n}, \varepsilon \in R^{m}
$$

Where, in the case of $\mathrm{m}=\mathrm{n}$, the transfer function $k$ is a lower triangular matrix, and $\mathrm{R}^{\mathrm{m} \times \mathrm{n}}$ represents a space having $\mathrm{m}$ rows and $\mathrm{n}$ columns.

In order to obtain $f$, equation (2) should be solved inversely to the established transfer function $\mathrm{k}$ and the known response $\varepsilon$. The solution (2) for $f$ is often referred to as deconvolution.

Usually, a simple method such as the standard inverse method, that is, a solution of $f=$ $\left(\mathrm{k}^{\mathrm{t}} \mathrm{k}\right)^{-1} \mathrm{k}^{\mathrm{t}} \varepsilon$ does not exist or if there is a result that may cause instability. Usually Tikhonov regularization is expressed as:

$$
\min \left\{\|k f-\varepsilon\|^{2}+\lambda\|I f\|^{2}\right\}
$$

However, the accuracy directly identified by the Tikhonov regularization method is not very high, so an improved regularization method specification must be adopted.

\subsection{Traditional Tikhonov regularization method}

Among the regularization methods, the Tikhonov regularization method is usually chosen to obtain stable solutions.

$$
\min \left\{\|\varepsilon-k f\|^{2}+\lambda\|I f\|^{2}\right\}
$$

Where $\lambda$ is the regularization parameter. Its regular solution is:

$$
f_{\lambda}=\left(k^{*} k+\lambda I\right)^{-1} k^{*} \varepsilon
$$

Where I is the identity matrix, $k^{*}$ is the conjugate of $k$. The part of the coefficient matrix at the right end of equation (5) adds the $\lambda I$ term, because this can play a role in overcoming the ill-conditioned matrix of the equation coefficient, so a stable solution can be obtained. Since the regular solution in (5) is related to the parameter $\lambda$, the value of $\lambda$ is different, and the solution is different. Therefore, how to choose the appropriate parameter $\lambda$ is the key to obtain a stable solution.

The traditional method uses the classical generalized cross validation method to select the appropriate parameter $\lambda$. Generalized cross validation is an effective method to select regularization parameter $\lambda$.

Cross validation is based on the statistics of the basic ideas, namely, an optimal regularization parameter value should make the model can predict the missing data well, the original data set is divided into training set and test set, firstly, the parameters are obtained by training set, and then the parameters are tested by test set, so that the parameter with the smallest test error is the optimal regularization parameter.

General cross validation depends on the order of data arrangement, while generalized cross validation is invariant for orthogonal transformation of data vectors. The advantage of the generalized cross-validation method is that it does not require prior information about the error norm. Therefore, the choice of the best regularization parameter $\lambda$ is required to make the GCV function $G(\lambda)$ obtain the minimum value. GCV function $G$ is defined as:

$$
G(\lambda)=\frac{\|k f-\varepsilon\|}{\left(\operatorname{trace}\left(I-k k_{\lambda}^{I}\right)\right)^{2}}
$$

Where, $f=k_{\lambda}^{I} \varepsilon$. Finally, the regularization parameter $\lambda$ obtained is input into equation (5) to solve the impact force. 


\section{Proposed Method}

\subsection{The homotopy function}

Because the Tikhonov regularization method has poor noise adaptability, and the L curve sometimes has multiple inflection points, resulting in underestimation [29]. In order to obtain a stable solution, this paper uses the regularization homotopy method. Suppose there are two topological spaces $\mathrm{X}$ and $\mathrm{Y}$ and two consecutive functions $f$ and $g$, and $f$ and $g$ are $\mathrm{X}$ to $\mathrm{Y}$ mappings, if there is a continuous mapping $\mathrm{H}:[0: 1] \mathrm{X} \rightarrow \mathrm{Y}$, Satisfied with the following formula:

$$
\forall x \in X, H(x, 0)=f(x) \quad \forall x \in X, H(x, 1)=g(x)
$$

Then the function $f$ and the function $g$ in the topological space $\mathrm{Y}$ are homotopy, and the function $\mathrm{H}$ is the path connecting $f$ and $g$. Therefore, if the equation $f(x)=0$ is to be solved, the homotopy idea can be used to construct the same function:

$$
H(\lambda, x)=(1-\lambda) f(x)+\lambda g(x)
$$

where $\lambda$ is expressed as a homotopy parameter, and when $\lambda$ is 0 , the equation is $f(x)=0$. Therefore, in the process of solving the equation, the value of $\lambda$ is continuously reduced in a certain way, and finally the solution of the original equation can be obtained.

Therefore, according to this homotopy idea, (8) is expressed as:

$$
\min =\varphi_{\lambda}(f)=\left\{(1-\lambda)\|k f-\varepsilon\|^{2}+\lambda\|f\|^{2}\right\}
$$

where $\lambda$ is a regularized homotopy parameter, which satisfies $0<\lambda<1$.

In order to minimize (9), the first derivative of $\lambda$ with respect to $f$ must be zero. Deriving (9) and letting its derivative equal to zero, you get:

$$
(1-\lambda) k^{*}(k f-\varepsilon)+\lambda f=0
$$

where the symbol $*$ indicates conjugate transposition. The impact force size can be expressed as:

$$
f_{\lambda}=\left[(1-\lambda I) k^{*} k+\lambda\right]^{-1}(1-\lambda) k^{*} \varepsilon
$$

\subsection{Newton downhill method}

Equation (11) is the regular solution of the improved regularization method. For the determination of the regularization parameter $\lambda$ in the formula, if the optimal regular solution is to be obtained, $\lambda$ should be gradually approached to zero. First, the Newton method is used to iteratively approach the minimum value. The steps are as follows:

Step 1: Let $\lambda_{t}$ be the root of $f_{\lambda}=0$, choose $\lambda_{0}$ as the initial approximation of $\lambda_{t}$, pass the point $\left(\lambda_{0}, f\left(\lambda_{0}\right)\right)$ as the tangent $\mathrm{L}$ of the curve $y=f(\lambda)$, and the equation of $\mathrm{L}$ is $\mathrm{y}=$ $f\left(\lambda_{0}\right)+f^{\prime}\left(\lambda_{0}\right)\left(\lambda-\lambda_{0}\right)$, find the intersection of $L$ and $x$ axis. The abscissa $\lambda_{1}=\lambda_{0}-$ $f\left(\lambda_{0}\right) / f^{\prime}\left(\lambda_{0}\right)$, said $\lambda_{1}$ is an approximation of $\lambda_{0}$.

Step 2: Over the point $\left(\lambda_{1}, f\left(\lambda_{1}\right)\right)$ to make the tangent of the curve $y=f(\lambda)$, and find the abscissa $\lambda_{2}=\lambda_{1}-f\left(\lambda_{1}\right) / f^{\prime}\left(\lambda_{1}\right)$ of the intersection of the tangent and the x-axis, and let $\lambda_{2}$ be the second approximation of $\lambda_{t}$.

Step 3: Repeat the above processes to obtain a sequence of $\lambda_{t}$ approximations, where $\lambda_{t+1}=\lambda_{t}-f\left(\lambda_{t}\right) / f^{\prime}\left(\lambda_{t}\right)$ is called the $t+1$ approximation of $\lambda_{t}$, and the above formula is called the Newton iteration formula.

The disadvantage of the Newton method is that its convergence depends on the selection of the initial value. If the initial value is far from the root of the root, the sequence obtained by Newton's method may diverge. To prevent iterative divergence, a condition is added to the 
iterative process to make it monotonic:

$$
\left|f\left(\lambda_{t+1}\right)\right|<\left|f\left(\lambda_{t}\right)\right|
$$

Then, the new improved value $\lambda_{t}$ is calculated by appropriately weighting the calculation result $\overline{\lambda_{\mathrm{t}+1}}=\lambda_{\mathrm{t}}-\frac{f\left(\lambda_{t}\right)}{f^{\prime}\left(\lambda_{t}\right)}$ of the above Newton method and the approximation $\lambda_{\mathrm{t}+1}=\alpha \overline{\lambda_{\mathrm{t}+1}}+$ $(1-\alpha) \lambda_{\mathrm{t}}$ of the previous step, where $\alpha(0<\alpha \leq 1)$ is the Newton downhill factor. The final calculation result is $\lambda_{\mathrm{t}+1}=\lambda_{\mathrm{t}}-\alpha \frac{f\left(\lambda_{\mathrm{t}}\right)}{f^{\prime}\left(\lambda_{\mathrm{t}}\right)}$.

When the Newton downhill factor is selected, it is halved from $\alpha=1$ until the condition of (12) is satisfied. The advantage of using Newton's downhill method is to ensure the parameters can be stably reduced. It also combines the fast convergence of Newton's method to achieve the purpose of this paper.

In this paper, the homotopy regularization parameters are defined between $[0,1]$. Therefore, the initial value of the regularization parameter is set to 1 , and then iteratively descends according to the Newton downhill iteration formula until it gradually approaches zero, and gets the most excellent regularization parameter.

\section{Experimental setup and process}

As shown in Fig. 1, the experimental equipment for the impact experiment of epoxy composite plate includes LC-01 impact hammer, NI USB-4431 data acquisition card, charge amplifier YE5852 and PZT sensor.

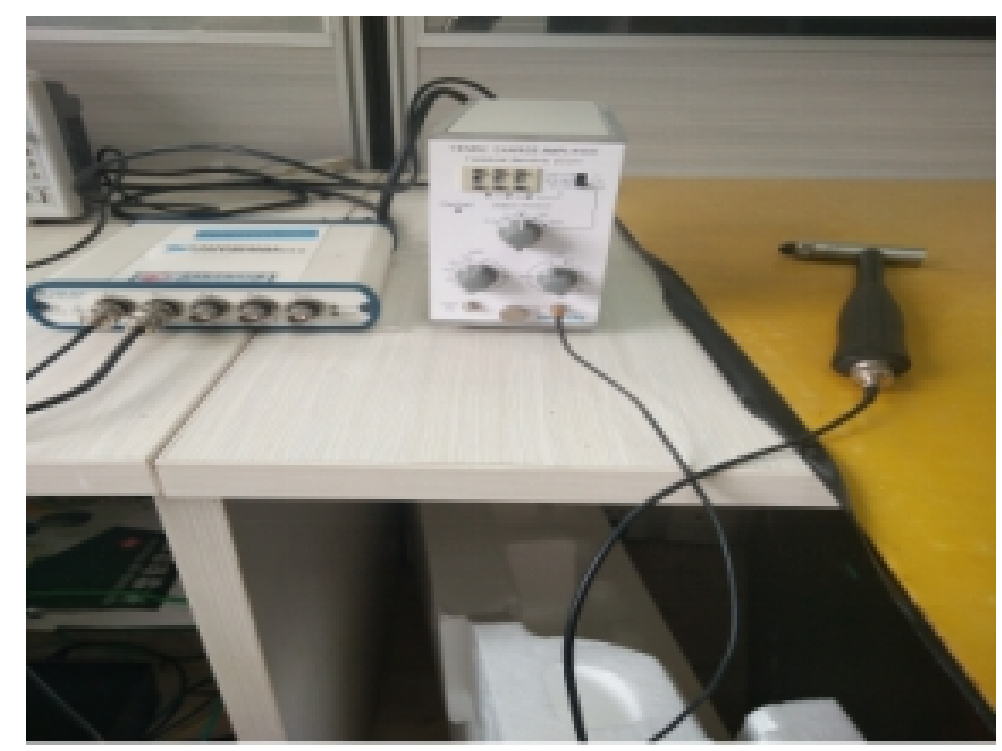

Fig. 1. Experimental set-up

Fig. 2 is a schematic diagram of the data acquisition system. The experimental system is divided into two parts: the hardware part and the software part. The hardware part includes data acquisition card, impact hammer containing IEPE (IEPE refers to a kind of acceleration sensor with power amplifier or voltage amplifier), charge amplifier, etc. The software includes 
Labview data acquisition system and MATLAB signal processing software.

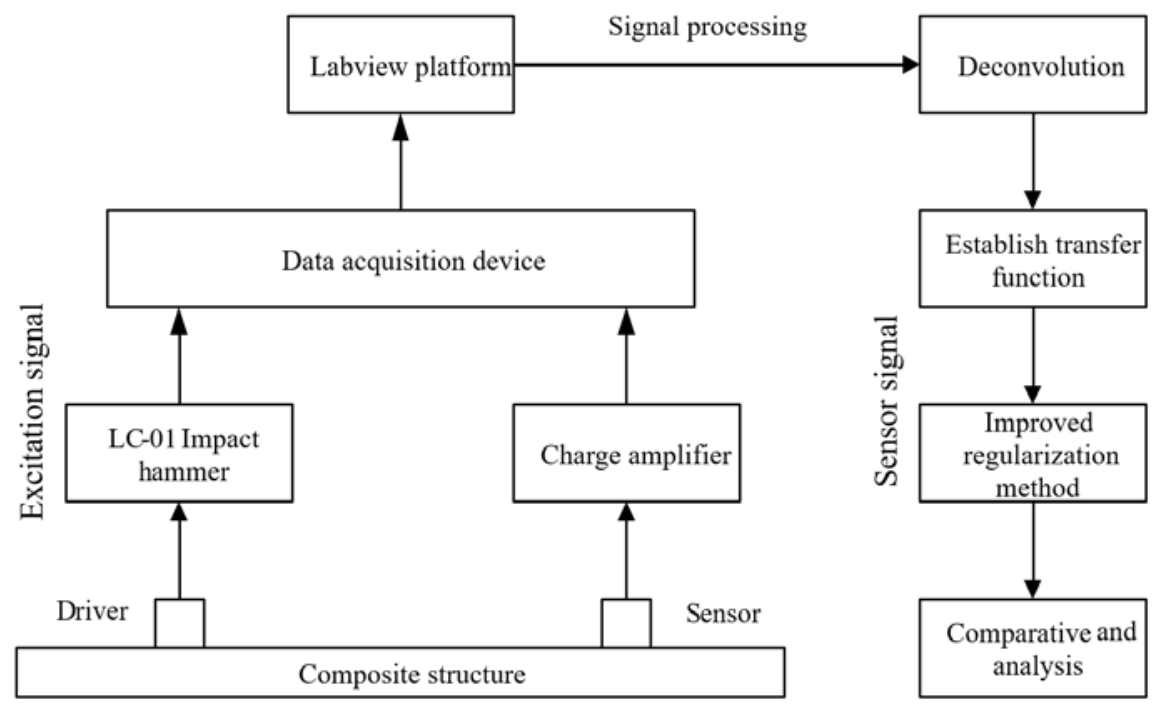

Fig. 2. Experimental process diagram

In the experiment, the PZT sensor and the impact hammer with built-in IEPE sensor were selected as the sensing element, and the NI USB-4431 data acquisition card was combined with the Labview software system to collect the impact signal caused by the impact hammer and simultaneously collect the vibration plate of the composite material plate. Since the output signal of the PZT sensor is very weak, a charge amplifier needs to be placed before receiving the piezoelectric sensing signal, the response signal to be acquired is amplified and converted in advance, and the response signal is collected by the data acquisition card. Since the data acquisition card can save the acquired impact signal and corresponding signal, the acquired signal can be processed and analyzed by MATLAB. Through the deconvolution, the transfer function is established, and then the regularization method is used to solve the ill-posed problem, and the optimal regular solution is obtained. Finally, the validity of the method is proved by comparing the error with the original impact force.

\section{Experimental Results and Analysis}

\subsection{Improved regularization method to reconstruct the impact force time history}

The criterion for judging the merits of the regularization parameter is whether the regularization parameter is infinitely approximately zero. As shown in Fig. 3, compared with the parameter of the traditional method, the improved regularization parameter is infinitely close to zero. Fig. 4 shows the time history comparison of the reconstructed impact force using the proposed method, the reconstructed impact force using the traditional method and the actual impact force. The experimental results show that the improved method reduces the noise interference to the experiment and is more stable. The improved impact force time history almost overlaps with the actual impact force time history. The actual impact force is $51.23 \mathrm{~N}$, and the improved impact force is 50.456N. Although the impact force of the traditional reconstruction method has little difference in size, the time history of reconstruction has a large deviation. 


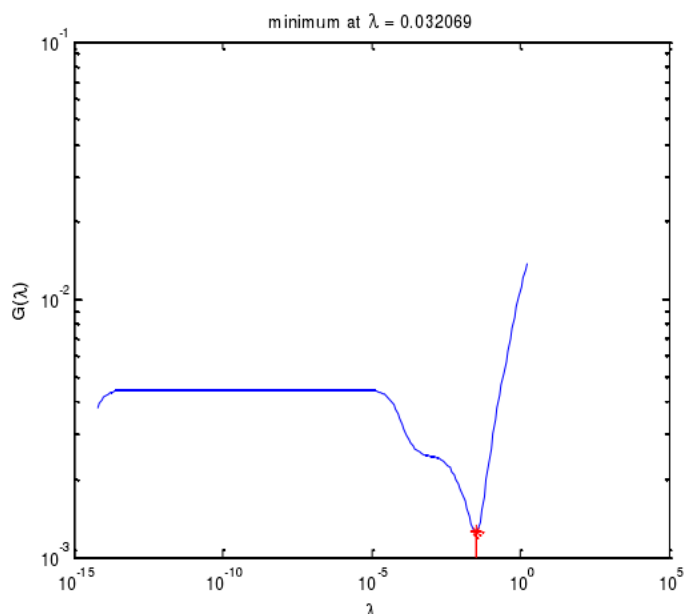

(a)

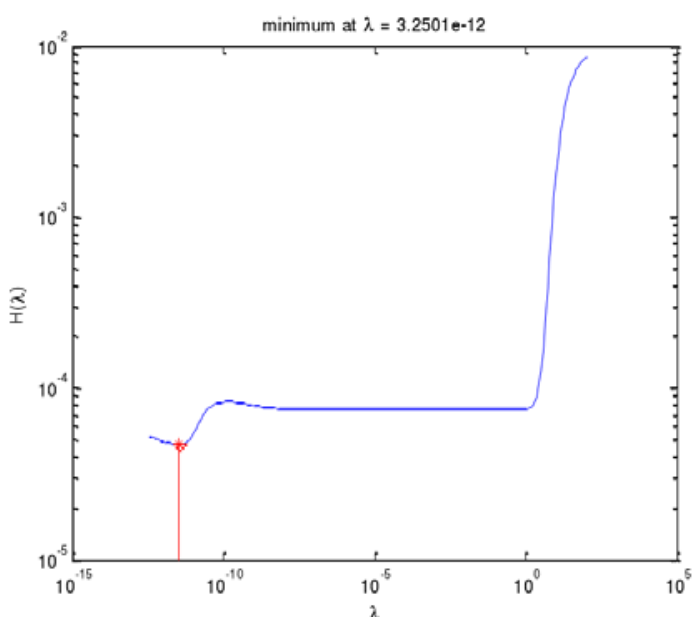

(b)

Fig. 3. Comparison of regularization parameters of two methods (a) Traditional regularization method, (b) Improved regularization method

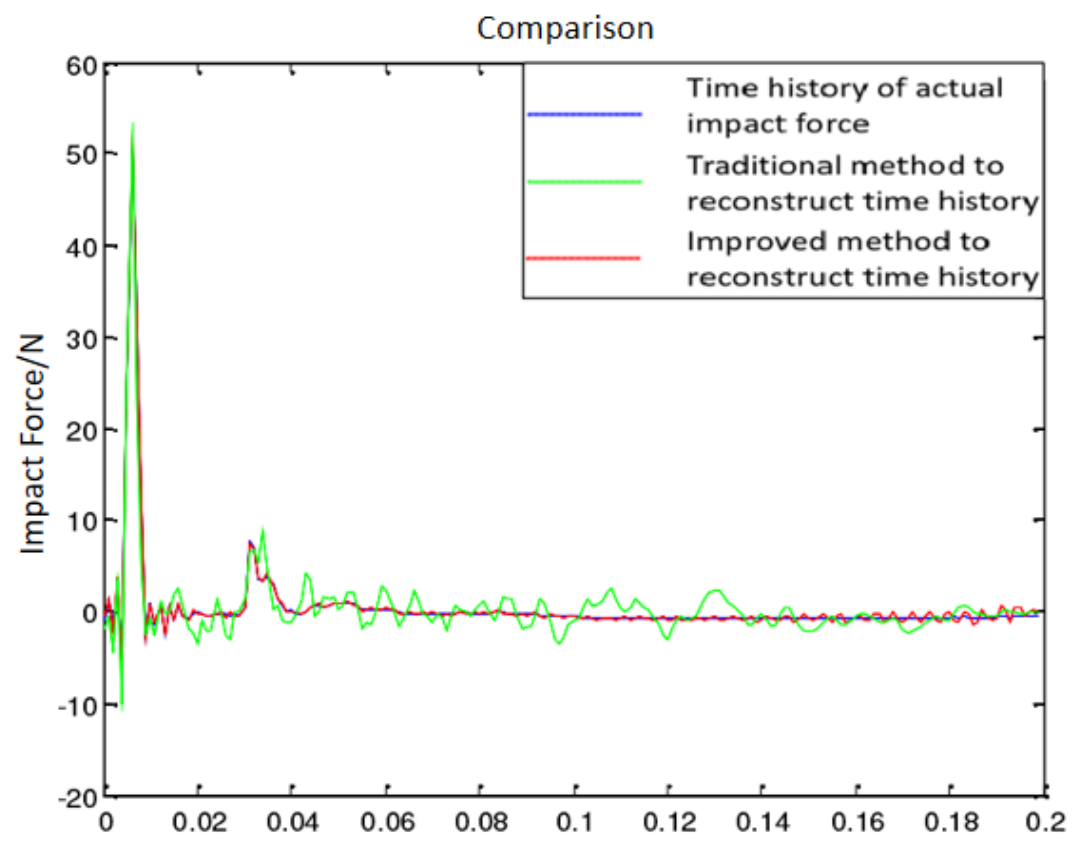

Fig. 4. Comparison of the time history between the two reconstruction methods and the actual impact force

In order to show the effectiveness of the proposed method, the absolute error $\delta$ and the relative error $\Delta$ are calculated separately.

$$
\begin{gathered}
\delta=\left|f_{1}-f\right| \\
\Delta=\frac{\left|f_{1}-f\right|}{f} \times 100 \%
\end{gathered}
$$


Table 1. The regularization parameters of the time history reconstruction of impact force by the two methods and the error comparison

\begin{tabular}{|c|c|c|c|}
\hline Class & $\begin{array}{c}\text { Regularization } \\
\text { parameter }\end{array}$ & Absolute error (N) & Relative error \\
\hline \hline $\begin{array}{c}\text { The traditional method to } \\
\text { reconstruct time history }\end{array}$ & 0.032069 & 8.9543 & 0.1336 \\
\hline $\begin{array}{c}\text { The improved method to } \\
\text { reconstruct time history }\end{array}$ & 0.000020 & 0.9774 & 0.0146 \\
\hline
\end{tabular}

The parameter comparison and error comparison of the recognition results are shown in Table 1. The regularization parameter of the traditional method is about 0.032 , while the improved regularization parameter is about 0.00002 . The absolute error of the traditional method is $8.9 \mathrm{~N}$, while the improved method has an absolute error of $8.9 \mathrm{~N}$. The absolute error is $0.97 \mathrm{~N}$, and the relative error in the final comparison is 0.133 in the traditional method, and only 0.01 in the improved method. Therefore, the improved method can get better applications.

\subsection{Reconstruction of impact force time history with different impact hammers}

The impact force on the composite plate is very sensitive to the location of the impact and the material properties of the impactor. This experiment uses different impact hammers to reconstruct the impact force time history (nylon hammer, aluminum hammer and stainless steel hammer). In Fig 5, from the overall comparison, it can be seen that the reconstruction results of different impact hammer improvement methods are better than the traditional method of impact force reconstruction results, and the error is very small. The results of the regularization parameters, absolute error and relative error of the three different impact hammers are shown in Table 2. The regularization parameters of each impact hammer improved method are close to zero, which is much smaller than the regularization parameters of the traditional reconstruction method. Moreover, the absolute error and relative error of the improved method are much smaller than that of the traditional reconstruction method. Therefore, the improved method of reconstructing the impact force time history in this paper is suitable for impact hammers of different stiffness.

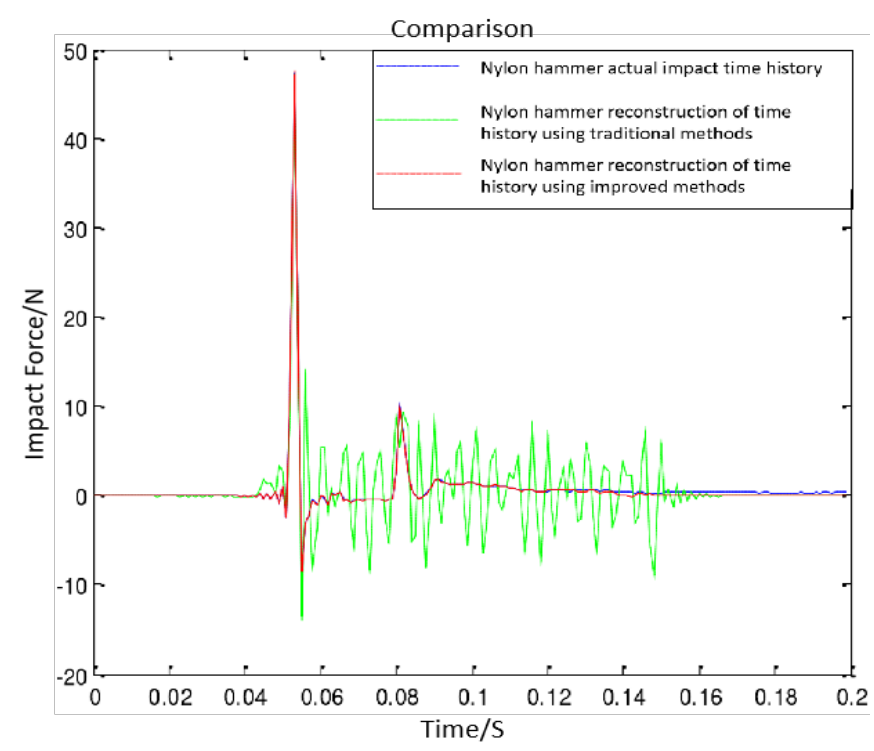

(a) 


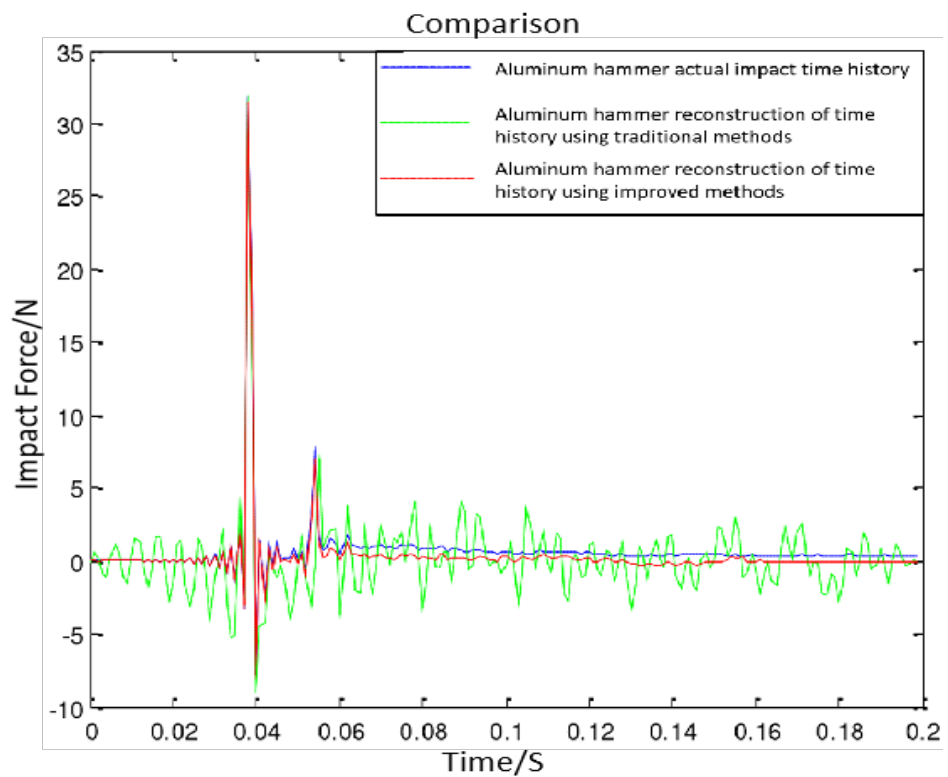

(b)

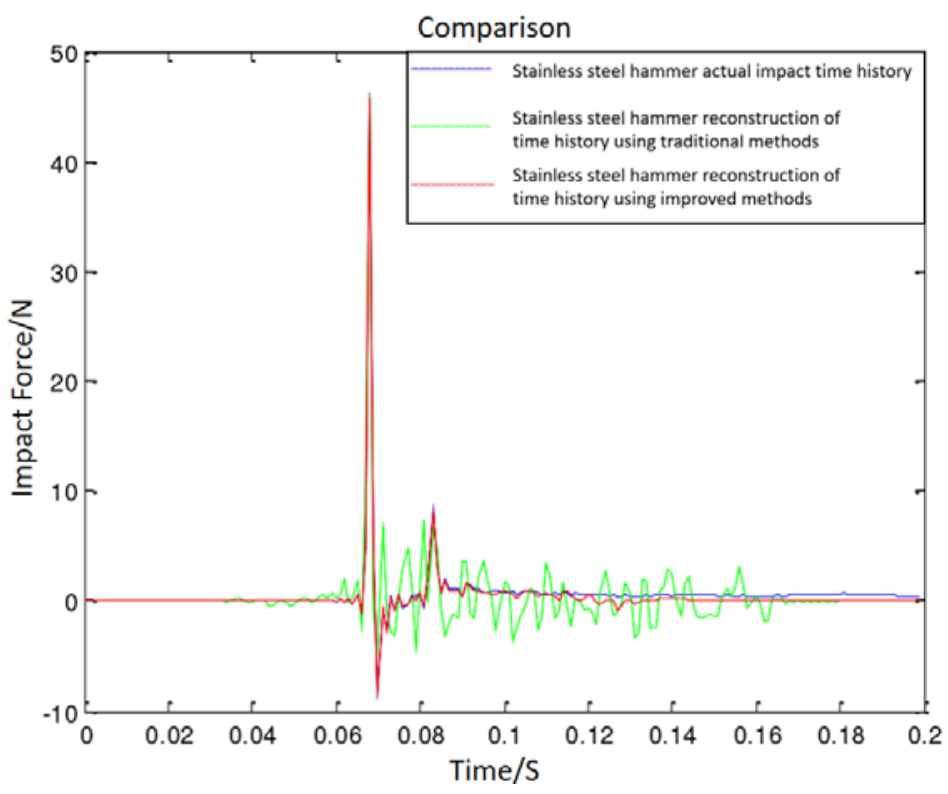

(c)

Fig. 5. Comparison of time history between impact force and actual impact force of different impact hammers reconstructed by two methods

(a) nylon hammer (b) aluminum hammer (c) stainless steel hammer 
Table 2. Two methods to reconstruct the regularization parameters and error comparison of the impact load time history of different impact hammers

\begin{tabular}{|c|c|c|c|}
\hline Class & $\begin{array}{c}\text { Regularization } \\
\text { parameter }\end{array}$ & Absolute error (N) & Relative error \\
\hline $\begin{array}{c}\text { The traditional method to } \\
\text { reconstruct the impact } \\
\text { time history of nylon } \\
\text { hammer }\end{array}$ & 0.058922 & 11.8956 & 0.3136 \\
\hline $\begin{array}{c}\text { The improved method to } \\
\text { reconstruct the impact } \\
\text { time history of nylon } \\
\text { hammer }\end{array}$ & 0.000342 & 0.4837 & 0.0090 \\
\hline $\begin{array}{c}\text { The traditional method to } \\
\text { reconstruct the impact } \\
\text { time history of aluminum } \\
\text { hammer }\end{array}$ & 0.344553 & 7.9959 & 0.1642 \\
\hline $\begin{array}{c}\text { The improved method to } \\
\text { reconstruct the impact } \\
\text { time history of aluminum } \\
\text { hammer }\end{array}$ & 0.000454 & 1.1980 & 0.0246 \\
\hline $\begin{array}{c}\text { The traditional method to } \\
\text { reconstruct the impact } \\
\text { time history of stainless } \\
\text { steel hammer }\end{array}$ & 0.323768 & 7.8845 & 0.2006 \\
\hline $\begin{array}{c}\text { The improved method to } \\
\text { reconstruct the impact } \\
\text { time history of stainless } \\
\text { steel hammer }\end{array}$ & 0.000442 & 0.8445 & 0.0215 \\
\hline
\end{tabular}

\section{Conclusion}

This paper introduces the regularization method for solving the ill-posed problem, and studies the advantages of the optimized regularization method for the time history reconstruction of the impact force. Firstly, the homotopy idea is introduced to the Tikhonov method, and the homotopy function is constructed. The Newton downhill method can be used to find the regularization parameter closest to zero, which is used for the homotopy function to obtain the optimal solution and accurately reconstruct the time history of the impact force. Under the same conditions, the time history and regularization parameters of the impact force were reconstructed by comparative experiments and unoptimized methods. Experiments show that with the optimization method, the time history accuracy of impact force reconstruction is significantly improved.

\section{References}

[1] N. W. Shi, "Experimental study on damage identification of cable-stayed Bridge model for health diagnosis,” Ph.D. dissertation, Dalian Maritime University, Dalian, China, 2017. Article(CrossRef Link)

[2] Y. J. Sun, and F. H. Gu, "Compressive sensing of piezoelectric sensor response signal for phased array structural health monitoring,” International Journal of Sensor Networks, vol. 23, no. 4, pp. 258-264, 2017. Article(CrossRef Link) 
[3] M. F. S. F. De. Moura, and A. T. Marques, "Prediction of low velocity impact damage in carbonepoxy laminates," Composites Part A: Applied Science and Manufacturing, vol. 33, no. 3, pp. 361368, 2002. Article(CrossRef Link)

[4] C. Y. Park, J. H. Kim, S. M. Jun, and C. G. Kim, "Localizations and force reconstruction of lowvelocity impact in a composite panel using optical fiber sensors," Advanced Composite Materials, vol. 21, no. 5-6, pp. 357-369, 2012. Article(CrossRef Link)

[5] L. Qiu, S. F. Yuan, H. F. Mei, and F. Fang, "An improved Gaussian mixture model for damage propagation monitoring of an aircraft wing spar under changing structural boundary conditions," Sensors, vol. 16, no. 3, pp. 291, 2016. Article(CrossRef Link)

[6] S. Y. Khooa, Z. Ismail, K. K. Kong, Z. C. Ong, S. Noroozi, W. T. Chong, and A. G. A. Rahman, "Impact force identification with pseudo-inverse method on a lightweight structure for underdetermined, even-determined and over-determined cases," International Journal of Impact Engineering, vol. 63, pp. 52-62, 2014. Article(CrossRef Link)

[7] C. Ma, and H. X. Hua, "The state space force identification technique based on an improved regularization method,” Journal Vibration and Shock, vol. 34, no. 11, pp. 146-149, 2015. Article(CrossRef Link)

[8] L. Wang, H. Cao, and Y. Xie, "An improved iterative Tikhonov regularization method for solving the dynamic load identification problem," International Journal for Computational Methods in Engineering Science and Mechanics, vol. 16, no. 5, pp. 292-300, 2015. Article(CrossRef Link)

[9] L. Guo, "Extreme learning machine with elastic net regularization," Intelligent Automation \& Soft Computing, vol. 26, no.3, pp. 421-427, 2020. Article(CrossRef Link)

[10] R. C. Aster, B. Borchers, and C. H. Thurber, "Discretizing Inverse Problems Using Basis Function” in Parameter estimation and inverse problems, 3sted., vol. 5, pp. 135-149, 2019. Article(CrossRef Link)

[11] A. E. Tümer, and A. Akkus, "Application of radial basis function networks with feature selection for gdp per capita estimation based on academic parameters," Computer Systems Science and Engineering, vol. 34, no.3, pp. 145-150, 2019. Article(CrossRef Link)

[12] L. J. Wang, X. Han, J. Liu, X. Q. He, and F. Huang, "A new regularization method and application to dynamic load identification problems," Inverse Problems in Science and Engineering, vol. 19, no. 6, pp. 765-776, 2011. Article(CrossRef Link)

[13] L. Qiu, S. F. Yuan, and W. B. Yang, "On research of time reversal imaging method and structural health monitoring system," International Journal of Applied Electromagnetics and Mechanics, vol. 46, no. 4, pp. 927-941, 2014. Article(CrossRef Link)

[14] C. L. Chen, Y. L. Li, and F. G. Yuan, "Impact source identification in finite isotropic plates using a time-reversal method : experimental study," Smart Materials and Structures, vol. 21, no.10, pp.105025, 2012. Article(CrossRef Link)

[15] S. Atobe1, H. Fukunaga, and N. Hu, "Impact Force Identification of CFRP Structures Using Experimental Transfer Matrices," Computers, Materials \& Continua, vol. 26, no. 1, pp. 67-90, 2011. Article(CrossRef Link)

[16] H. Kalhori, L. Ye, S. Mustapha, J. Li, and B. Li, "Reconstruction and Analysis of Impact Forces on a Steel-Beam-Reinforced Concrete Deck,” Experimental Mechanics, vol. 56, pp. 1547-1558, 2016. Article(CrossRef Link)

[17] H. Kalhori, L. Ye, and S. Mustapha, "Inverse estimation of impact force on a composite panel using a single piezoelectric sensor," Journal of Intelligent Material Systems and Structures, vol. 28, no. 6, pp.799-810, 2017. Article(CrossRef Link)

[18] C. S. Liu, "A dynamical Tikhonov regularization method for solving nonlinear ill-posed linear algebraic systems,” Acta Applicandae Mathematicae, vol. 123, pp. 285-307, 2013. Article(CrossRef Link)

[19] J. Li, X.L Li, B. Yang, and X.M. Sun, "Segmentation-based Image Copy-move Forgery Detection Scheme," IEEE Transactions on Information Forensics and Security, vol. 10, no. 3, pp. 507-518, 2015. Article(CrossRef Link) 
[20] S. Mustapha, and L. Ye, "Propagation behaviour of guided waves in tapered sandwich structures and debonding identification using time reversal,” Wave Motion, vol. 57, pp. 154-170, 2015. Article(CrossRef Link)

[21] S. Mustapha, L. Ye, X. J. Dong, and M. M. Alamdari, "Evaluation of barely visible indentation damage (BVID) in CF/EP sandwich composites using guided wave signals,” Mechanical Systems and Signal Processing, vol. 76-77, pp. 497-517, 2016. Article(CrossRef Link)

[22] M. Rezghi, and S. M. Hosseini, “A new variant of L-curve for Tikhonov regularization,” Journal of computational and applied mathematics, vol. 231, no. 2, pp. 914-924, 2009. Article(CrossRef Link)

[23] C. S. Liu, H. K. Hong, and S. N. Atluri, "Novel algorithms based on the conjugate gradient method for inverting ill-conditioned matrices, and a new regularization method to solve ill-posed linear systems," Computer Modeling in Engineering and Sciences, vol. 60, pp. 279-308, 2010. Article(CrossRef Link)

[24] X. P. Wang, J. Cai, and Z. Q. Zhou, "A signal domain transform method for spatial resolution improvement of Lamb wave signals with synthetically measured relative wavenumber curves," Structural Health Monitoring, vol. 18, no. 5-6, pp. 1633-1651, 2018. Article(CrossRef Link)

[25] X. P. Wang, J. Cai, and Z. Q. Zhou, “A Lamb wave signal reconstruction method for highresolution damage imaging,” Chinese Journal of Aeronautics, vol. 32, no. 5, pp. 1087-1099, 2019. Article(CrossRef Link)

[26] J. W. Zhang, Z. Qin, and S. F. Wang, “A new adaptive regularization parameter selection based on expected patch log likelihood,” Journal of Cyber Security, vol. 2, no. 1, pp. 25-36, 2020. Article(CrossRef Link)

[27] L. J. Wang, Y. Huang, Y. X. Xie, and Y. X.Du, “A new regularization Method for dynamic load identification,” Science Progress, vol. 103, no. 3, pp.1-15, 2020. Article(CrossRef Link)

[28] J. Lu, and G. Fei, "Non-linear localization algorithm based on newton iterations,” Journal of Internet of Things, vol. 2, no. 4, pp. 129-134, 2020. Article(CrossRef Link)

[29] C. S. Liu, "Optimally generalized regularization methods for solving linear inverse problems," Computers, Materials \& Continua, vol. 29, no. 2, pp.103-128, 2012. Article (CrossRef Link) 


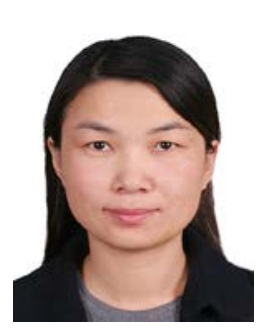

Yajie Sun is currently an associate professor in Nanjing University of Information Science and Technology, Nanjing, China. She received Ph.D. degree in test measurement technology and instrument engineering from Nanjing University of Aeronautics and Astronautics. Her research interests include structural health monitoring, signal processing and big data.

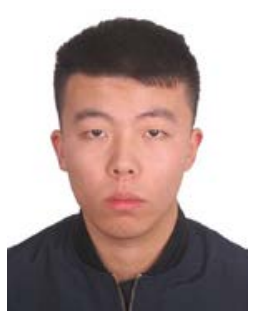

Tao Yin received the B.S. degree ininternet of things engineering from Nanjing University of Information Science and Technology, China, in 2019. He is now pursuing a M.S. degree in software engineering at Nanjing University of Information Science \& Technology, Nanjing, JiangSu, China. His research interest is composite material structure health monitoring.

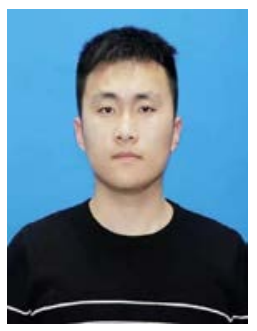

Jian Yang received the B.S. degree in Electronic Information Engineering from Jiangsu Ocean University. China,in2019. He is now pursuing a M.S. degree in software engineering at Nanjing University of Information Science\& Technology, Nanjing, JiangSu, China.His research interest is composite material structure health monitoring.

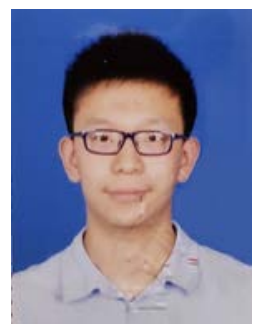

Zhiyu Cai, currently studying as an undergraduate at Nanjing University of Information Science \& Technology, is now majoring in information security. His research interest is Deep learning convolutional neural network.

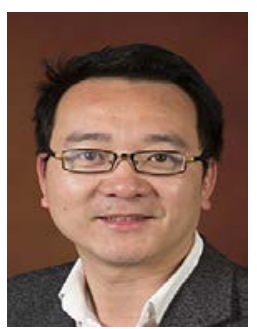

Shaoen Wu received his $\mathrm{PhD}$ degree in Computer Science and Software Engineering from Auburn University, He serves on the Advisory Council of Scholarship for the Vice Provost for Research, and the Dean's Faculty Advisory Board. He is a senior member of IEEE, the secretary of IEEE MMTC 2018-2020, and a member of ACM. He has published over 70 peerreviewed papers in wireless, IoT, smart health and robotics at international journals e.g. IEEE Internet of Things Journal and conferences e.g. IEEE Globecom, ICC and ICCCN. 